\title{
关于正弦级数 $L^{1}$ - 收敛性研究中对单调递减条件 的确切推广
}

\section{周颂平}

浙江理工大学数学研究所, 杭州 310018

E-mail: songping.zhou@163.com

收稿日期: 2010-02-05; 接受日期: 2010-04-13

摘要 本文在处理 $L^{1}$ - 收敛性问题中给出了一个确切的条件和一种更直接的方式.

关键词 单调性 有界变差 正弦级数 收玫性

MSC (2000) 主题分类 $42 \mathrm{~A} 20,42 \mathrm{~A} 32$

0 引言

记 $L_{2 \pi}$ 为具有 $2 \pi$ 周期的可积函数空间. 记

$$
g(x):=\sum_{n=1}^{\infty} a_{n} \sin n x, \quad f(x):=\sum_{n=1}^{\infty} a_{n} \cos n x .
$$

如常, 记 $S_{n}(g, x)$ 为正弦级数 $g$ 的 $n$ 阶部分和, 即

$$
S_{n}(g, x)=\sum_{k=1}^{n} a_{k} \sin k x,
$$

此外 $\|\cdot\|_{L^{1}}=\int_{0}^{2 \pi}|\cdot| d x$.

在经典 Fourier 分析领域中, 三角级数的可积性问题一直被认为是一个有趣但困难的课题, 这实 际上等价于确认一个三角级数是否是一个 Fourier 级数, 因此形成了绝大部分进一步研究包括收玫性 研究的原始基础. 例如, 在研究 $L^{1}$ - 收玫性和逼近度的问题中, 人们一般总是需要一个先决的假设 $f \in L_{2 \pi}$. 从这个观点来看, 上述可积性问题等同于对系数数列 $\left\{a_{n}\right\}$ 设置何种合适条件可以保证 $f(x)$ 与 $g(x)$ 的可积性的问题.

20 世纪 50 年代, Boas ${ }^{[1]}$ 以及 Heywood ${ }^{[2]}$ 证明了如下结果:

定理 $\mathbf{B H}$ 设 $a_{n} \searrow 0$. 对于 $0 \leqslant \alpha<2$,

$$
g(x) / x^{\alpha} \in L_{2 \pi} \Leftrightarrow \sum_{n=1}^{\infty} n^{\alpha-1} a_{n}<\infty
$$

对于 $0<\alpha<1$,

$$
f(x) / x^{\alpha} \in L_{2 \pi} \Leftrightarrow \sum_{n=1}^{\infty} n^{\alpha-1} a_{n}<\infty
$$


以后出现过一些对于加权可积性 (即, 对于不等式 (0.2) 以及条件 $0<\alpha<2$ 下的不等式 (0.1)) 的 关于系数数列 $\left\{a_{n}\right\}$ 非负单调递减条件的推广工作, 可参见文献 [3-8]. 事实上, 单调递减条件的推广 从各种拟单调条件和有界变差条件最后发展到了本质上是最终条件的均值有界变差条件, 有兴趣的读 者可以参考文献 [9] 以找到有用的信息. 这里我们仅仅给出与本文内容有关的一些条件的定义.

定义 1 非负数列 $\mathrm{A}=\left\{a_{n}\right\}_{n=1}^{\infty}$ 称为是拟单调的, 是指对于某一 $\alpha \geqslant 0$, 数列 $\left\{a_{n} / n^{\alpha}\right\}_{n=1}^{\infty}$ 是单 调递减的, 记作 $\mathrm{A} \in \mathrm{QMS}$ (Quasi-Monotone Sequences).

定义 2 如果对某一 $O$ - 正则变化数列 $\{R(n)\}_{n=1}^{\infty}$, 数列 $\mathrm{A}=\left\{a_{n} / R(n)\right\}_{n=1}^{\infty}$ 是非负单调递减 的, 则称数列 $\left\{a_{n} / R(n)\right\}_{n=1}^{\infty}$ 是 $O-$ 正则变化拟单调的, 而 $O$ - 正则变化是指数列 $\{R(n)\}_{n=1}^{\infty}$ 是一个正 的递增数列并且有 $\limsup _{n \rightarrow \infty} \frac{R(2 n)}{R(n)}<\infty$. 此时, 记 A $\in$ RQMS (Regulaly-Varying Quasi-Monotone Sequences).

定义 $3^{[10]}$ 如果正的趋向于零的数列 $\mathrm{A}=\left\{a_{n}\right\}_{n=1}^{\infty}$ 对所有自然数 $n$ 满足

$$
\sum_{k=n}^{\infty}\left|a_{k}-a_{k+1}\right|=: \sum_{k=n}^{\infty}\left|\Delta a_{k}\right| \leqslant M(\mathrm{~A}) a_{n},
$$

则称数列 $\mathrm{A}$ 为 “剩余有界变差数列”, 简记为 $\mathrm{A} \in$ RBVS (Rest Bounded Variation Sequences).

定义 $4^{[11]}$ 如果非负数列 $\mathrm{A}=\left\{a_{n}\right\}_{n=1}^{\infty}$ 对所有自然数 $n$ 满足

$$
\sum_{k=n}^{2 n}\left|\Delta a_{k}\right| \leqslant M(\mathrm{~A}) a_{n}
$$

则称数列 $\mathrm{A}$ 为 “分组有界变差数列”, 简记为 $\mathrm{A} \in$ GBVS (Group Bounded Variation Sequences).

两年后, Tikhonov 也在 [7] 中提出 GBVS, 并称之为 “广义单调数列” (General Monotone Sequences).

定义 $5^{[9]}$ 如果非负数列 $\mathrm{A}=\left\{a_{n}\right\}_{n=1}^{\infty}$ 对所有自然数 $n$ 和某个 $\lambda \geqslant 2$ 满足

$$
\sum_{k=n}^{2 n}\left|\Delta a_{k}\right| \leqslant \frac{M(\mathrm{~A})}{n} \sum_{k=[n / \lambda]}^{[\lambda n]} a_{k}
$$

其中 $[x]$ 表示不超过 $x$ 的最大整数, 则称数列 $\mathrm{A}$ 为 “均值有界变差数列”, 简记为 $\mathrm{A} \in$ MVBVS (Mean Value Bounded Variation Sequences).

我们同时使用 MS 表示通常所有非负单调 (递减) 数列的集合.

这些集合之间的关系可简略描述如下:

$$
\begin{aligned}
& \mathrm{MS} \varsubsetneqq \mathrm{QMS} \varsubsetneqq \mathrm{RQMS}, \quad \mathrm{MS} \varsubsetneqq \mathrm{RBVS}, \\
& \mathrm{QMS} \nsubseteq \mathrm{RBVS}, \operatorname{RBVS} \nsubseteq \mathrm{QMS} \quad \text { (见 [5]), } \\
& \mathrm{RBVS} \cup \mathrm{RQMS} \varsubsetneqq \mathrm{GBVS} \quad(\text { 见 }[11]), \\
& \text { GBVS } \varsubsetneqq \operatorname{MVBVS} \quad \text { (见 [9]), }
\end{aligned}
$$

概括起来,

$$
\text { MS } \varsubsetneqq \text { RBVS } \cup \text { QMS } \varsubsetneqq \text { RBVS } \cup \text { RQMS } \varsubsetneqq \text { GBVS } \varsubsetneqq \text { MVBVS }
$$

总而言之, MVBVS 是本质性推广单调 (递减) 数列的最大集合之一, 并在 Fourier 分析各种经典 的收玫性问题中得到了广泛而重要的应用 (参见 [9]). 
此外, 还有一些对于 MVBVS 的推广工作 (参见 $[12,13]$ ), 但由于它们与本文的内容无关而不在此 涉及.

现在让我们回顾最近一个关于加权 $L^{1}$ - 可积性的结果 (见 [8]).

定理 WZ1 设 $\left\{a_{n}\right\} \in$ MVBVS. 对于 $0<\alpha<2$,

$$
g(x) / x^{\alpha} \in L_{2 \pi} \Leftrightarrow \sum_{n=1}^{\infty} n^{\alpha-1} a_{n}<\infty ;
$$

对于 $0<\alpha<1$,

$$
f(x) / x^{\alpha} \in L_{2 \pi} \Leftrightarrow \sum_{n=1}^{\infty} n^{\alpha-1} a_{n}<\infty .
$$

然而, 最重要的真正的可积性问题 (即当 $\alpha=0$ 时的关系式 (0.1)) 在几十年里差不多无人涉及. 直到最近, [8] 才给出了这方面的一个尝试, 但其结论与平常的加权情形有很大的差别:

定理 WZ2 设 $\left\{a_{n}\right\} \in \operatorname{MVBVS}$, 那么, 如果 $g(x) \in L_{2 \pi}$, 我们有 $\sum_{n=1}^{\infty} n^{-1} a_{n}<\infty$. 另一方面, 存 在一个数列 $\left\{a_{n}\right\} \in \mathrm{MVBVS}$ 使得 $\sum_{n=1}^{\infty} n^{-1} a_{n}<\infty$ 成立, 但是正弦级数 $g(x)=\sum_{n=1}^{\infty} a_{n} \sin n x$ 不属 于空间 $L_{2 \pi}$.

之后, 我们发现, 如果直接考虑 $g$ 的不定积分, 容易证明以下的

命题 如果 $g(x) \in L_{2 \pi}$, 那么 $\left\{a_{n}\right\}$ 是其 Fourier 系数, 并且 $\sum_{n=1}^{\infty} n^{-1} a_{n}$ 收玫.

这样定理 WZ2 的前一部分则成为上述命题的一个推论.

我们现在换一种角度来思考问题.

给定一个正弦级数 $\sum_{n=1}^{\infty} a_{n} \sin n x$, 在收玫点 $x$ 处其和函数记为 $g(x)$. 如上所言, 去验证这个和 函数或正弦级数本身是否可积是一个很难的任务. 另一方面, 在研究三角级数的 $L^{1}$ - 收玫性时, 人们 又需要假设一个先决条件即级数是可积的, 例如, 周知的 $L^{1}$ - 收玫性定理告诉我们 (参见 [14]), 设实的 偶 (奇) 函数 $f \in L_{2 \pi}$, 其 Fourier 余弦 (正弦) 系数 $\left\{a_{n}\right\}_{n=1}^{\infty}$ 单调递减, 则 $\lim _{n \rightarrow \infty}\left\|f-S_{n}(f)\right\|_{L^{1}}=0$ 的充分必要条件是 $\lim _{n \rightarrow \infty} a_{n} \log n=0$. 同时, 关于正弦级数一致收玫性的经典 Chaudy-Jolliffe 定理 给出了以下的另一种形式: 设 $\left\{a_{n}\right\}_{n=1}^{\infty}$ 单调递减, 那么正弦级数 $\sum_{n=1}^{\infty} a_{n} \sin n x$ 一致收玫的充分必要 条件是 $\lim _{n \rightarrow \infty} n a_{n}=0$. 这两个经典定理之间的差别很明显: 一个需要先决的可积性条件, 而另一个 则无需先决的连续性条件. 数学家显然青睐于后一种类型的定理, 但在 $L^{1}$ - 收玫性定理中难以避免这 个先决条件的原因也正是积分计算的复杂性和艰巨性导致的, 而且设立了这个先决条件可以很容易地 将计算级数绝对值的积分 (这是一个无限的过程) 转化为计算有限和绝对值的积分. 还可以进一步看 出, 在可积空间, 首要的问题应该是 $L^{1}$ - 收玫性问题, 这可能通过直接验证级数本身的系数而获得, 理 论上并不需要涉及其和函数.

基于以上原因, 为什么我们不去尝试找到一个 $L^{1}$ - 收玫性定理的更直接和明晰的形式呢? 事实上, 容易用类似的方法证明以上提到的 Boas-Heywood 经典定理 (定理 BH 中当 $\alpha=0$ 时的关系式 (0.1)) 对 $L^{1}$ - 收敛性具有以下的等价形式:

定理 $\mathbf{B H}^{*}$ 给定一个正弦级数 $\sum_{n=1}^{\infty} a_{n} \sin n x$, 在收玫点 $x$ 处其和函数记为 $g(x)$. 设 $\left\{a_{n}\right\}$ 是非 负单调递减数列并且 $\lim _{n \rightarrow 0} a_{n}=0$, 那么 $\lim _{n \rightarrow \infty}\left\|g-S_{n}(g)\right\|_{L^{1}}=0$ 的充分必要条件是

$$
\sum_{n=1}^{\infty} n^{-1} a_{n}<\infty
$$

这里, 在任何情形下, $\lim _{n \rightarrow \infty}\left\|g-S_{n}(g)\right\|_{L^{1}}=0$ 理解为其 Cauchy 收敛性准则 $\left\|S_{n}(g)-S_{m}(g)\right\|_{L^{1}}$ $\rightarrow 0, n, m \rightarrow \infty$ 成立, 反之亦然. 
从这个思路出发, 我们开始本文的研究工作.

总体上, 将本篇论文的结构做如下安排. 首先, 在第二节, 我们构造反例说明通常的单调性条件的 推广即使是拟单调性也不足以保证以上经典定理 (定理 $\mathrm{BH}^{*}$ ) 的结论成立 (定理 1). 然后, 在第 2 节, 我们提出一个正确的新条件能够取代单调性条件, 推广了上面的经典定理 (定理 2), 而这个条件包容 了很多前人提出的条件 (定理 3 和 4). 最后, 我们证明了, 为保证 $L^{1}$ - 收玫性经典结论成立, 这个条件 本质上已不能再进一步减弱 (定理 5 ).

贯穿全文, 我们总使用 $M(x)$ 或者 $C(x)$ 来表示一个仅依赖于 $x$ 的正常数, 其中 $x$ 可以是变量或 者数列, 而用 $C$ 来表示一个正的绝对常数, 它们的值在不同的场合未必相同. 但有时在特定的地方, 为 了区别常数的需要, 我们也使用不同的符号, 例如 $C_{1}, C_{2}$ 等去表示它们.

\section{1 一个反例}

本节我们建立

定理 1 存在一个非负数列 $\left\{a_{n}\right\} \in$ QMS 使得 $\sum_{n=1}^{\infty} \frac{a_{n}}{n}<\infty$, 但是对于正弦级数

$$
g(x):=\sum_{n=1}^{\infty} a_{n} \sin n x
$$

有 $\left\|g-S_{n}(g)\right\|_{L^{1}} \nrightarrow 0, \quad n \rightarrow \infty$.

证明 置 $n_{1}=1, n_{2}=2, m_{j}=2^{j^{2}}, n_{j}=j^{2} 2^{j^{2}}, j=3,4, \ldots$ 令 $a_{1}, \ldots, a_{3}$ 为任意给定的正数, 对于 $j \geqslant 2$, 令

$$
a_{n}= \begin{cases}\frac{n}{m_{j+1} \log ^{2} n}, & 2 n_{j} \leqslant n<m_{j+1}, \\ \frac{n}{m_{j+1} \log ^{2} n_{j+1}}, & m_{j+1} \leqslant n<n_{j+1}, \\ \frac{1}{2 \log n_{j+1}}, & n_{j+1} \leqslant n<2 n_{j+1} .\end{cases}
$$

如此我们已经定义了一个正弦级数并记在其收玫点 $x$ 处, $g(x):=\sum_{n=1}^{\infty} a_{n} \sin n x$.

不太困难, 可以验证 $\left\{a_{n}\right\} \in$ QMS. 事实上, 不失一般性, 我们仅需要考虑对充分大的 $n$, 验证 $\left\{a_{n} / n\right\}$ 是一个单调递减数列. 从表达式

$$
\frac{a_{n}}{n}= \begin{cases}\frac{1}{m_{j+1} \log ^{2} n}, & 2 n_{j} \leqslant n<m_{j+1}, \\ \frac{1}{m_{j+1} \log ^{2} n_{j+1}}, & m_{j+1} \leqslant n<n_{j+1}, \\ \frac{1}{2 n \log n_{j+1}}, & n_{j+1} \leqslant n<2 n_{j+1}\end{cases}
$$

出发, 并注意到 $n_{k} \geqslant m_{k} \log m_{k}$, 以及对于充分大的 $k, 2 \log m_{k}=2 k^{2} \log 2 \geqslant \log k+k^{2} \log 2=\log n_{k}$, 从而

$$
\frac{a_{n_{j+1}}}{n_{j+1}}=\frac{1}{2 n_{j+1} \log n_{j+1}} \leqslant \frac{1}{2 m_{j+1} \log m_{j+1} \log n_{j+1}} \leqslant \frac{1}{m_{j+1} \log ^{2} n_{j+1}}=\frac{a_{n_{j+1}-1}}{n_{j+1}-1},
$$

我们清楚地看到 $a_{n} / n$ 对于 $2 n_{j} \leqslant n<2 n_{j+1}$ 是单调递减的. 同时,

$$
\frac{a_{2 n_{j+1}}}{2 n_{j+1}}=\frac{1}{m_{j+2} \log ^{2}\left(2 n_{j+1}\right)} \leqslant \frac{1}{2\left(2 n_{j+1}-1\right) \log n_{j+1}}=\frac{a_{2 n_{j+1}-1}}{2 n_{j+1}-1}
$$

804 
也是明显的. 所有上述讨论显示对于充分大的 $n,\left\{a_{n} / n\right\}$ 是单调递减的, 即 $\left\{a_{n}\right\} \in$ QMS.

其次, 我们计算得 (注意 $n_{k+1} \leqslant C m_{k+1} \log n_{k+1}$ )

$$
\begin{aligned}
\sum_{n=2 n_{2}}^{\infty} \frac{a_{n}}{n} & =\sum_{k=2}^{\infty} \sum_{j=2 n_{k}}^{2 n_{k+1}-1} \frac{a_{j}}{j}=\sum_{k=2}^{\infty}\left(\sum_{j=2 n_{k}}^{m_{k+1}-1}+\sum_{j=m_{k+1}}^{n_{k+1}-1}+\sum_{n_{k+1}}^{2 n_{k+1}-1}\right) \frac{a_{j}}{j} \\
& =\sum_{k=2}^{\infty} \sum_{j=2 n_{k}}^{m_{k+1}-1} \frac{1}{m_{k+1} \log ^{2} j}+\sum_{k=2}^{\infty} \sum_{j=m_{k+1}}^{n_{k+1}-1} \frac{1}{m_{k+1} \log ^{2} n_{k+1}}+\sum_{k=2}^{\infty} \sum_{j=n_{k+1}}^{2 n_{k+1}-1} \frac{1}{2 j \log n_{k+1}} \\
& \leqslant \sum_{k=2 n_{2}}^{\infty} \frac{1}{j \log ^{2} j}+\sum_{k=n_{2}}^{\infty} \frac{n_{k+1}}{m_{k+1} \log ^{2} n_{k+1}}+\sum_{k=n_{2}}^{\infty} \frac{C}{\log n_{k+1}} \\
& \leqslant \sum_{k=2 n_{2}}^{\infty} \frac{1}{j \log ^{2} j}+\sum_{k=n_{2}}^{\infty} \frac{C}{(k+1)^{2}}<\infty .
\end{aligned}
$$

记

$$
\phi_{n}(x):=\sum_{k=1}^{n}\left(\frac{\sin (n+k) x}{k}-\frac{\sin (n-k) x}{k}\right)=2 \cos n x \sum_{k=1}^{n} \frac{\sin k x}{k},
$$

应用熟知的不等式

$$
\sup _{n \geqslant 1} \max _{-\infty<x<\infty}\left|\sum_{k=1}^{n} \frac{\sin k x}{k}\right| \leqslant 3 \sqrt{\pi}
$$

得到

$$
\int_{0}^{2 \pi} \phi_{n_{k+1}}(x)\left(S_{2 n_{k+1}}(g, x)-S_{n_{k+1}}(g, x)\right) d x \leqslant C \int_{0}^{2 \pi}\left|S_{2 n_{k+1}}(g, x)-S_{n_{k+1}}(g, x)\right| d x,
$$

而对于 $k \geqslant 2$,

$$
\int_{0}^{2 \pi} \phi_{n_{k+1}}(x)\left(S_{2 n_{k+1}}(g, x)-S_{n_{k+1}}(g, x)\right) d x=\sum_{j=1}^{n_{k+1}} \frac{a_{n_{k+1}+j}}{j} \geqslant \frac{1}{2 \log n_{k+1}} \sum_{j=1}^{n_{k+1}-1} \frac{1}{j} \geqslant C>0,
$$

联系 (2), 我们就有

$$
\int_{0}^{2 \pi}\left|S_{2 n_{k+1}}(g, x)-S_{n_{k+1}}(g, x)\right| d x \geqslant C,
$$

因此 $\left\|g-S_{n}(g)\right\|_{L^{1}} \nrightarrow 0, n \rightarrow \infty$. 证明完毕.

\section{2 正弦级数的 $L^{1}$ - 收敛性}

我们现在研究正弦级数的 $L^{1}$ - 收敛性并证明

定理 2 设 $\left\{a_{n}\right\}$ 是一个非负有界数列. 在收玫点 $x$ 处, 记 $g(x)=\sum_{n=1}^{\infty} a_{n} \sin n x$. 给定一个整数 $N>0$, 置

$$
R_{n}=\sum_{k=n}^{\infty}\left|\Delta \frac{a_{k}}{\log ^{N} k}\right|
$$

对于所有 $n=1,2, \ldots$, 假设

$$
R_{n} \leqslant M(\mathrm{~A}) \frac{a_{n}}{\log ^{N} n}
$$


则 $\lim _{n \rightarrow \infty}\left\|g-S_{n}(g)\right\|_{L^{1}}=0$ 的充分必要条件是 $\sum_{n=1}^{\infty} \frac{a_{n}}{n}<\infty$.

证明 必要性是引言所提出命题的直接推论. 我们证明定理的充分性. 任意给定充分大的 $m, n$, 比如说 $m>n$, 记

$$
S_{m}(x)-S_{n}(x)=\sum_{k=n+1}^{m} a_{k} \sin k x=\sum_{k=n+1}^{m} \frac{a_{k}}{\log ^{N} k} \log ^{N} k \sin k x .
$$

由 Abel 变换,

$$
\begin{aligned}
S_{m}(x)-S_{n}(x)= & -\frac{a_{n+1}}{\log ^{N}(n+1)} \sum_{j=1}^{n} \log ^{N} j \sin j x+\frac{a_{m}}{\log ^{N} m} \sum_{j=1}^{m} \log ^{N} j \sin j x \\
& +\sum_{k=n+1}^{m-1} \Delta \frac{a_{k}}{\log ^{N} k} \sum_{j=1}^{k} \log ^{N} j \sin j x \\
=: & I_{1}(x)+I_{2}(x)+I_{3}(x) .
\end{aligned}
$$

我们计算

$$
\sum_{j=1}^{k} \log ^{N} j \sin j x=\sum_{j=1}^{k-1} \Delta \log ^{N} j \sum_{l=1}^{j} \sin l x+\log ^{N} k \sum_{l=1}^{k} \sin l x
$$

因此

$$
\int_{0}^{\pi}\left|\sum_{j=1}^{k} \log ^{N} j \sin j x\right| d x \leqslant \sum_{j=1}^{k-1}\left|\Delta \log ^{N} j\right| \int_{0}^{\pi}\left|\sum_{l=1}^{j} \sin l x\right| d x+\log ^{N} k \int_{0}^{\pi}\left|\sum_{l=1}^{k} \sin l x\right| d x \leqslant C \log ^{N+1} k,
$$

这样得到

$$
\int_{0}^{\pi}\left|I_{1}(x)\right| d x \leqslant C \frac{a_{n+1}}{\log ^{N}(n+1)} \log ^{N+1} n \leqslant C a_{n+1} \log n,
$$

以及

$$
\int_{0}^{\pi}\left|I_{2}(x)\right| d x \leqslant C a_{m} \log m,
$$

同时还有

$$
\begin{aligned}
\int_{0}^{\pi}\left|I_{3}(x)\right| d x & \leqslant \sum_{k=n+1}^{m-1}\left|\Delta \frac{a_{k}}{\log ^{N} k}\right| \int_{0}^{\pi}\left|\sum_{j=1}^{k} \log ^{N} j \sin j x\right| d x \\
& \leqslant \sum_{k=n+1}^{m-1}\left|\Delta \frac{a_{k}}{\log ^{N} k}\right| \log ^{N+1} k=: J .
\end{aligned}
$$

应用条件 (3), 进一步的研究得到

$$
\begin{aligned}
J & =\sum_{k=n+1}^{m-1}\left(R_{k}-R_{k+1}\right) \log ^{N+1} k \\
& =\left|R_{n+1} \log ^{N+1}(n+1)-R_{m} \log ^{N+1} m+\sum_{j=n+1}^{m-1} R_{j+1}\left(\log ^{N+1}(j+1)-\log ^{N+1} j\right)\right| \\
& \leqslant C_{N}\left(a_{n+1} \log (n+1)+a_{m} \log (m+1)+\sum_{j=n+1}^{m-1} \frac{a_{j+1}}{j+1}\right)
\end{aligned}
$$


此处我们已经再次应用了 Abel 变换.

因为 (3) 成立, 对于 $[\sqrt{n}] \leqslant j \leqslant n$ 我们看出

$$
\begin{aligned}
\frac{a_{n}}{\log ^{N} n} & =-\sum_{k=j}^{n-1} \Delta \frac{a_{k}}{\log ^{N} j}+\frac{a_{j}}{\log ^{N} j} \\
& \leqslant \sum_{k=j}^{\infty}\left|\Delta \frac{a_{k}}{\log ^{N} j}\right|+\frac{a_{j}}{\log ^{N} j} \\
& \leqslant(M(\mathrm{~A})+1) \frac{a_{j}}{\log ^{N} j},
\end{aligned}
$$

于是对所有 $[\sqrt{n}] \leqslant j \leqslant n, a_{n} \leqslant C a_{j}$ 成立. 因此,

$$
a_{n+1} \log n \leqslant C a_{n} \sum_{j=[\sqrt{n}]}^{n} \frac{1}{j} \leqslant C \sum_{j=[\sqrt{n}]}^{n} \frac{a_{j}}{j}, \quad a_{m} \log m \leqslant C \sum_{j=[\sqrt{m}]}^{m} \frac{a_{j}}{j} .
$$

综合所有估计 (4)-(8), 结合 Cauchy 收玫准则, 我们最终证明了所需要的结论.

如果一个非负有界数列 $\left\{a_{n}\right\}$ 满足条件 (3), 我们称其为一个对数剩余有界变差数列 (Logarithm Rest Bounded Variation Sequence), 记作 $\left\{a_{n}\right\} \in \operatorname{LRBVS}_{N}$.

类似地, 一个非负数列 $\left\{a_{n}\right\}$ 被称为对数拟单调数列, 如果对于某个正整数 $N,\left\{a_{n} / \log ^{N} n\right\}$ 单调 递减, 这时记作 $\left\{a_{n}\right\} \in \mathrm{LQMS}_{N}$.

下一个定理显示了有关集合之间的关系, 其中特别重要的是 RBVS 是 $\mathrm{LRBVS}_{N}$ 的真子集的结论.

定理 3 对于任何整数 $N \geqslant 2$, 成立

$$
\text { RBVS } \cup \mathrm{LQMS} \varsubsetneqq \mathrm{LRBVS}_{1} \varsubsetneqq \mathrm{LRBVS}_{2} \varsubsetneqq \cdots \varsubsetneqq \operatorname{LRBVS}_{N} .
$$

证明 关系式

$$
\text { RBVS } \subset \mathrm{LRBVS}_{1}, \quad \text { LQMS } \varsubsetneqq \mathrm{LRBVS}_{1}
$$

是清楚的. 我们只需要证明 RBVS $\neq \mathrm{LRBVS}_{1}$. 置 $n_{1}=1$, 对于 $j \geqslant 2, n_{j}=2^{2^{j^{2}}}$. 定义 $a_{1}, a_{2}, \ldots, a_{n_{2}-1}$ 为任意给定的正数,

$$
a_{n}=\frac{\log n}{\log ^{3} n_{j+1}}, \quad n_{j} \leqslant n<n_{j+1}, \quad j=2,3, \ldots
$$

因为

$$
\frac{a_{n}}{\log n}=\frac{1}{\log ^{3} n_{j+1}}, \quad n_{j} \leqslant n<n_{j+1}, \quad j=2,3, \ldots,
$$

显然 $a_{n} / \log n$ 对于 $j \geqslant 2$ 是单调递减的, 因而 $\left\{a_{n}\right\} \in \mathrm{LRBVS}_{1}$. 另一方面,

$$
\sum_{k=n_{j}}^{n_{j+1}-1}\left|\Delta a_{k}\right|=\frac{1}{\log n_{j+1}^{2}}-\frac{\log n_{j}}{\log ^{3} n_{j+1}},
$$

于是

$$
\frac{\sum_{k=n_{j}}^{\infty}\left|\Delta a_{k}\right|}{a_{n_{j}}} \geqslant \frac{\sum_{k=n_{j}}^{n_{j+1}-1}\left|\Delta a_{k}\right|}{a_{n_{j}}} \geqslant C \frac{\log n_{j+1}}{\log n_{j}} \geqslant C \frac{2^{(j+1)^{2}}}{2^{j^{2}}}
$$

即 $\left\{a_{n}\right\} \notin$ RBVS.

这里, 我们顺便注意到显然有 $\sum_{n=1}^{\infty} a_{n} / n<\infty$. 
对于 $N \geqslant 2$, 其它的关系式

$$
\operatorname{LRBVS}_{1} \varsubsetneqq \operatorname{LRBVS}_{2} \varsubsetneqq \cdots \varsubsetneqq \operatorname{LRBVS}_{N}
$$

可以类似地证明.

推论 1 对于某个整数 $N>0$, 设 $\left\{a_{n}\right\} \in \mathrm{LQMS}_{N}, g(x)$ 为定理 2 中所定义的正弦级数, 那么 $\left\|g-S_{n}(g)\right\|_{L^{1}}=0$ 的充分必要条件是

$$
\sum_{n=1}^{\infty} \frac{a_{n}}{n}<\infty
$$

推论 2 设 $\left\{a_{n}\right\} \in \mathrm{RBVS}, g(x)$ 为定理 2 中所定义的正弦级数, 那么 $\left\|g-S_{n}(g)\right\|_{L^{1}}=0$ 的充分 必要条件是

$$
\sum_{n=1}^{\infty} \frac{a_{n}}{n}<\infty
$$

注记 1 在上面所有结果中, $L^{1}$ - 收玫性的结论不能由通常的 $L^{1}$ - 收玫定理直接推出, 因为此时 $g \in L_{2 \pi}$ 的先决条件并不假定满足.

在经典情形下, 当 $\left\{a_{n}\right\} \in \mathrm{MS}$ 时, 人们早已知道

$$
\sum_{n=1}^{\infty} \frac{a_{n}}{n}<\infty
$$

与

$$
\sum_{n=1}^{\infty}\left|\Delta a_{n}\right| \log n<\infty
$$

是等价的. 现在我们证明当 $\left\{a_{n}\right\} \in \mathrm{LRBVS}_{N}$ 时, (9) 与 (10) 也是等价的.

定理 4 设 $\left\{a_{n}\right\} \in \operatorname{LRBVS}_{N}$, 那么条件

$$
\sum_{n=1}^{\infty} \frac{a_{n}}{n}<\infty
$$

与

$$
\sum_{n=1}^{\infty}\left|\Delta a_{n}\right| \log n<\infty
$$

是等价的.

证明 应用 Abel 变换, 有

$$
\sum_{k=1}^{n} \frac{a_{k}}{k}=\sum_{k=1}^{n-1} \Delta a_{k} \sum_{j=1}^{k} \frac{1}{j}+a_{n} \sum_{j=1}^{n} \frac{1}{j},
$$

这样

$$
\sum_{k=1}^{n} \frac{a_{k}}{k} \leqslant \sum_{k=1}^{n-1}\left|\Delta a_{k}\right| \log k+a_{n} \log n .
$$

由于

$$
a_{n} \log n \leqslant \log n \sum_{k=n}^{\infty}\left|\Delta a_{k}\right| \leqslant \sum_{k=n}^{\infty}\left|\Delta a_{k}\right| \log k
$$

808 
从条件 (10) 推出 (9) 是很容易的. 另一方面, 注意到

$$
a_{k}-a_{k+1}=\log ^{N}(k+1) \Delta \frac{a_{k}}{\log ^{N} k}-\frac{a_{k}}{\log ^{N} k}\left(\log ^{N}(k+1)-\log ^{N} k\right),
$$

我们获得

$$
\sum_{k=1}^{n}\left|\Delta a_{k}\right| \log k \leqslant \sum_{k=1}^{n}\left|\Delta \frac{a_{k}}{\log ^{N} k}\right| \log ^{N+1}(k+1)+\sum_{k=1}^{n} \frac{a_{k}}{\log ^{N} k}\left(\log ^{N}(k+1)-\log ^{N} k\right) \log k .
$$

与定理 2 证明中处理 $J$ 的情形类似, 我们有

$$
\sum_{k=1}^{n}\left|\Delta \frac{a_{k}}{\log ^{N} k}\right| \log ^{N+1}(k+1) \leqslant C_{N}\left(a_{n+1} \log (n+1)+\sum_{k=1}^{n} \frac{a_{k}}{j}\right) .
$$

同时

$$
\sum_{k=1}^{n} \frac{a_{k}}{\log ^{N} k}\left(\log ^{N}(k+1)-\log ^{N} k\right) \log k \leqslant C \sum_{k=1}^{n} \frac{a_{k}}{\log ^{N} k} \frac{\log ^{N-1} k}{k} \log k \leqslant C \sum_{k=1}^{n} \frac{a_{k}}{k},
$$

结合 (11) 与定理 2 证明中的 (8), 我们最终获得

$$
\sum_{k=1}^{n}\left|\Delta a_{k}\right| \log k \leqslant C_{N} \sum_{k=1}^{n} \frac{a_{k}}{j},
$$

因此完成了定理 4 的证明.

\section{3 条件的最终性}

下面的定理显示, 为保证 $L^{1}$ - 收玫性经典结论成立, 这个新提出的条件本质上已不能再进一步 减弱.

定理 5 设 $R(n)$ 是一个满足 $\lim _{n \rightarrow \infty} R(n)=\infty$ 以及 $\lim _{\sup } \operatorname{su}_{n \rightarrow \infty} \frac{R(2 n)}{R(n)}<\infty$ 的单调递增数列. 如果

$$
\sum_{k=1}^{n} \frac{R(k)}{k} \leqslant C R(n), \quad n=1,2, \ldots
$$

则存在一个正数列 $\left\{a_{n}\right\}$ 使得 $a_{n} / R(n)$ 单调递减而且满足

$$
\sum_{n=1}^{\infty} \frac{a_{n}}{n}<\infty
$$

但是, 对于正弦级数

$$
g(x)=\sum_{n=1}^{\infty} a_{n} \sin n x
$$

则有 $\left\|g-S_{n}(g)\right\|_{L^{1}} \nrightarrow 0, n \rightarrow \infty$.

证明 证明的基本思路与定理 1 相似. 置 $n_{1}=1, n_{2}=2$, 下面归纳选择 $n_{j}$. 对于 $j \geqslant 2$, 选择了 $n_{j}$ 后, 令

$$
m_{j+1}=2^{n_{j}^{2}} .
$$


由 (12), 我们看到

$$
C R(n) \geqslant \sum_{k=[\sqrt{n}]}^{n} \frac{R(k)}{k} \geqslant C_{1} R([\sqrt{n}]) \log n,
$$

从而

$$
\frac{R(n)}{\log n} \geqslant C_{2} R([\sqrt{n}]) \rightarrow \infty, \quad n \rightarrow \infty
$$

这样取

$$
n_{k+1}=\min \left\{n \geqslant m_{k+1}: \frac{R(n)}{\log n} \geqslant R\left(m_{k+1}\right)\right\} .
$$

很明显, 从定义看, $n_{k+1}>m_{k+1}$, 并且有

$$
\frac{R\left(n_{k+1}-1\right)}{\log \left(n_{k+1}-1\right)} \leqslant R\left(m_{k+1}\right) .
$$

现在定义

$$
a_{n}= \begin{cases}\frac{R(n)}{R\left(m_{j+1}\right) \log ^{2} n}, & 2 n_{j} \leqslant n<m_{j+1}, \\ \frac{R(n)}{R\left(m_{j+1}\right) \log ^{2} n_{j+1}}, & m_{j+1} \leqslant n<n_{j+1}, \\ \frac{1}{\log n_{j+1}}, & n_{j+1} \leqslant n<2 n_{j+1} .\end{cases}
$$

由

结合 (13), 我们计算得

$$
\frac{a_{n}}{R(n)}= \begin{cases}\frac{1}{R\left(m_{j+1}\right) \log ^{2} n}, & 2 n_{j} \leqslant n<m_{j+1}, \\ \frac{1}{R\left(m_{j+1}\right) \log ^{2} n_{j+1}}, & m_{j+1} \leqslant n<n_{j+1}, \\ \frac{1}{R(n) \log n_{j+1}}, & n_{j+1} \leqslant n<2 n_{j+1},\end{cases}
$$

$$
\frac{a_{n_{j+1}}}{R\left(n_{j+1}\right)}=\frac{1}{R\left(n_{j+1}\right) \log n_{j+1}} \leqslant \frac{1}{R\left(m_{j+1}\right) \log ^{2} n_{j+1}}=a_{n_{j+1}-1},
$$

顾及其它显然的事实, 我们清楚地看出 $a_{n} / R(n)$ 是单调递减的.

关于 $\sum_{n=1}^{\infty} a_{n} / n<\infty$ 的估计也类似于定理 1 的证明, 联系 (12) 与 (14), 我们即有

$$
\begin{aligned}
\sum_{n=2 n_{2}}^{\infty} \frac{a_{n}}{n} & =\sum_{k=2}^{\infty} \sum_{j=2 n_{k}}^{2 n_{k+1}-1} \frac{a_{j}}{j}=\sum_{k=2}^{\infty}\left(\sum_{j=2 n_{k}}^{m_{k+1}-1}+\sum_{j=m_{k+1}}^{n_{k+1}-1}+\sum_{n_{k+1}}^{2 n_{k+1}-1}\right) \frac{a_{j}}{j} \\
& =\sum_{k=2}^{\infty} \sum_{j=2 n_{k}}^{m_{k+1}-1} \frac{R(j)}{j R\left(m_{k+1}\right) \log ^{2} j}+\sum_{k=2}^{\infty} \sum_{j=m_{k+1}}^{n_{k+1}-1} \frac{R(j)}{j R\left(m_{k+1}\right) \log ^{2} n_{k+1}}+\sum_{k=2}^{\infty} \sum_{j=n_{k+1}}^{2 n_{k+1}-1} \frac{1}{j \log n_{k+1}} \\
& \leqslant \sum_{k=2 n_{2}}^{\infty} \frac{1}{j \log ^{2} j}+\sum_{k=n_{2}}^{\infty} \frac{R\left(n_{k+1}-1\right)}{R\left(m_{k+1}\right) \log ^{2} n_{k+1}}+\sum_{k=n_{2}}^{\infty} \frac{C}{\log n_{k+1}} \\
& \leqslant \sum_{k=2 n_{2}}^{\infty} \frac{1}{j \log ^{2} j}+\sum_{k=n_{2}}^{\infty} \frac{C}{n_{k}^{2}}<\infty .
\end{aligned}
$$


最后, 完全复制定理 1 的证明, 我们获得

$$
\int_{0}^{2 \pi}\left|S_{2 n_{k+1}}(x)-S_{n_{k+1}}(x)\right| d x \geqslant C,
$$

这样我们就得到了所需的结论. 证明完毕.

注记 2 除了如 $\left\{\log ^{N} n\right\}$ 那样的 “缓慢增长” 数列以外, 其它 “非缓慢增长” 数列 (例如 $\left\{n^{\alpha}\right\}$, $\alpha>0$ 等) 显然满足条件 (12). 因此, 在某种意义上, 定理 5 说明 LRBV 条件对正弦级数的 $L^{1}$ - 收玫性 已不能进一步减弱.

\author{
参考文献 \\ 1 Boas Jr R P. Integrability of trigonometric series. J Math Oxford Ser, 1952, 3: 217-221 \\ 2 Heywood P. On the integrability of functions defined by trigonometric series. Quart J Math Oxford Ser, 1954, 5: 71-76 \\ 3 Askey R, Wainger S. Integrability theorems for Fourier series. Duke Math J, 1966, 33: 223-228 \\ 4 Boas Jr R P. Integrability Theorems for Trigonometric Transforms. New York: Springer, 1967 \\ 5 Leindler L. A new class of numerical sequences and its applications to sine and cosine series. Anal Math, 2002, 28: \\ 279-286 \\ 6 Tikhonov S. On the integrability of trigonometric series. Math Notes, 2005, 78: 437-442 \\ 7 Tikhonov S. Trigonometric series with general monotone coefficients. J Math Anal Appl, 2007, 326: 721-735 \\ 8 Wang M Z, Zhou S P. Applications of MVBV condition in $L^{1}$-integrability. Acta Math Hungar, to appear \\ 9 Zhou S P, Zhou P, Yu D S. Ultimate generalization for monotonicity for uniform convergence of trigonometric series. \\ Sci China Math, 2010, 53: 1853-1862 \\ 10 Leindler L. On the uniform convergence and boundedness of a certain class of sine series. Anal Math, 2001, 27: 279-285 \\ 11 Le R J, Zhou S P. A new condition for the uniform convergence of certain trigonometric series. Acta Math Hungar, \\ 2005, 108: 161-169 \\ 12 Tikhonov S. On $L^{1}$-convergence of Fourier series. J Math Math Appl, 2008, 347: 416-427 \\ 13 Korus P. Remarks on the uniform and $L^{1}$-convergence of trigonometric series. Acta Math Hungar, to appear \\ 14 Zygmund A. Trigonometric Series. Cambridge: Cambridge University Press, 1977
}

\title{
What condition can correctly generalize monotonicity in $L^{1}$-con- vergence of sine series?
}

\section{ZHOU SongPing}

Abstract In classical Fourier analysis, the integrability of trigonometric series is considered as an interesting but difficult topic. In particular, the integrability of sine series have not been touched in dozens years since Boas, Heywood published their classical results, meanwhile the generalizations of (deceasing) monotonicity have been developed from various quasimonotonicity and bounded variation conditions, finally, to the mean value bounded variation condition, an essential ultimate condition in most sense, and applied to various convergence problems extensively including uniform convergence, mean convergence, $L^{p}$ integrability and best approximation etc. The difficulty of the research can be seen from this point.

We may need another point of view now. Given a sine series $\sum_{n=1}^{\infty} a_{n} \sin n x$, its sum function can be written as $g(x)$ at the point $x$ where it converges. However, it is usually a very hard job to verify if the sum function or the sine series itself belonging to $L_{2 \pi}$ or not. On the other hand, in studying $L^{1}$-convergence problems, people usually need a requirement that $g \in L_{2 \pi}$, which also becomes a hard condition to check or a prior condition to set in most cases. For instance, the well-known classical results for $L^{1}$-convergence says that, let the real even (odd) function $f \in L_{2 \pi}$, and its Fourier cosine (sine) coefficients $\left\{a_{n}\right\}_{n=1}^{\infty} \in M S$, then $\lim _{n \rightarrow \infty}\left\|f-S_{n}(f)\right\|_{L^{1}}=0$ if and only if $\lim _{n \rightarrow \infty} a_{n} \log n=0$. On the other hand, the classical result of uniform convergence of sine series 
(Chaudy-Jolliffe theorem) says that, let $\left\{a_{n}\right\}_{n=1}^{\infty} \in M S$, then the sine series $\sum_{n=1}^{\infty} a_{n} \sin n x$ uniformly converges if and only if $\lim _{n \rightarrow \infty} n a_{n}=0$. The difference of two types of classical theorems is very clear: one need a prior condition $f \in L_{2 \pi}$, the other does not require that $f \in C_{2 \pi}$. Mathematicians surely prefer the latter to the former in mathematical sense. The reason that the prior condition in $L^{1}$ case cannot be avoided mainly arises from the much more "computation complexity" in the integrable space than the continuous space, and technical benefit to turn the computation from "infinity" into "finiteness" by setting the integrability. Furthermore, people also note that the first important problem in the integrable space should be $L^{1}$-convergence, which may be achieved by the series itself (by coefficients) without mentioning the sum function.

Based upon these reasons, why do we not try to find a more direct and clean version of $L^{1}$-convergence? The present paper is arranged as follows. First construct a nonnegative sequence which shows that although it is quasimonotone and the inequality $\sum_{n=1}^{\infty} n^{-1} a_{n}<\infty$ holds, but the corresponding sine series does not converge in $L^{1}$-norm (Theorem 1). Then, we raised a new correct condition which can guarantee the above mentioned condition to be necessary and sufficient for which the corresponding sine series does converge in $L^{1}$ norm (Theorem 2), and also discuss the relationships with other related sets (Theorems 3 and 4). Finally, we prove the newly raised condition cannot be weakened further in this $L^{1}$-convergence case in some sense (Theorem 5). As a whole, we give a complete solution to this topic.

Keywords: trigonometric series, convergence, integrability, monotonicity

$\operatorname{MSC}(2000): \quad 42 \mathrm{~A} 25,42 \mathrm{~A} 50$ 